Sammlung Metzler

Band 336 
Dennis F. Mahoney

\section{Friedrich von Hardenberg (Novalis)}

Verlag J.B. Metzler Stuttgart · Weimar 


\section{Der Autor}

Dennis F. Mahoney, geb. 1950; Studium der Germanistik, Philosophie und Geschichte in Amherst, Massachusetts und Freiburg; 1977 Promotion; Professor für Germanistik und Direktor des Programms für Europäische Studien an der University of Vermont (USA); zahlreiche Aufsätze zur Literatur zwischen 1750 und 1850 und zum deutschen Film. Bei J.B. Metzler ist erschienen: Der Roman der Goethezeit. SM 241. 1988.

Die Deutsche Bibliothek - CIP-Einheitsaufnahme

Mahoney, Dennis F:

Friedrich von Hardenberg (Novalis) / Dennis F. Mahoney.

- Stuttgart ; Weimar : Metzler, 2001

(Sammlung Metzler ; Bd. 336)

ISBN 978-3-476-10336-9

SM 336

ISBN 978-3-476-10336-9

ISBN 978-3-476-05164-6 (eBook)

DOI $10.1007 / 978-3-476-05164-6$

ISSN 05583667

Dieses Werk einschließlich aller seiner Teile ist urheberrechtlich geschützt. Jede Verwertung außerhalb der engen Grenzen des Urheberrechtsgesetzes ist ohne Zustimmung des Verlages unzulässig und strafbar. Das gilt insbesondere für Vervielfältigungen, Übersetzungen, Mikroverfilmungen und die Einspeicherung und Verarbeitung in elektronischen Systemen.

(c) 2001 Springer-Verlag GmbH Deutschland

Ursprünglich erschienen bei J.B. Metzlersche Verlagsbuchhandlung und Carl Ernst Poeschel Verlag GmbH in Stuttgart 2001

www.metzlerverlag.de

info@metzlerverlag.de 


\section{Vorwort}

Seit der Veröffentlichung von Novalis Schriften im Jahr 1802 und der biographischen Einleitung zur dritten Auflage von 1815 hat der Mythos, den Friedrich Schlegel und Ludwig Tieck damit aufbauten, nicht nur ein Verständnis des damals noch weitgehend unveröffentlichten Werkes verhindert, sondern auch den Zugang zum dahinter verborgenen Autor erschwert. Tiecks Darstellung zufolge habe sein Freund "nur wenige Dichter gelesen" und sei in der Poesie, der Kritik und den Systemen der Dichtkunst "eigentlich eben so Fremdling" gewesen, wie er sich auf Erden seit dem Tod seiner ersten Braut betrachtet habe:

"Ihm war es zur natürlichsten Ansicht geworden, das Gewöhnlichste, Nächste als ein Wunder, und das Fremde, Uebernatürliche als etwas Gewöhnliches zu betrachten, so umgab ihn das alltägliche Leben selbst wie ein wundervolles Märchen, und jene Region, die die meisten Menschen nur als ein Fernes, Unbegreifliches ahnden oder bezweifeln wollen, war ihm wie eine liebe Heimath. So erfand er, von Beispielen unbestochen, einen neuen Weg der Darstellung, und in der Vielseitigkeit der Beziehung, in der Ansicht der Liebe und dem Glauben an sie, die ihm zugleich Lehrerinn, Weisheit und Religion ist, darin daß ein einziger großer Lebens-Moment und Ein tiefer Schmerz und Verlust das Wesen seiner Poesie und Anschauung wurde, gleicht er unter den Neueren allein dem erhabenen Dante, und singt uns wie dieser einen unergründlichen mystischen Gesang [...] « (IV, 559).

So wurde "Novalis« zum Inbegriff der Romantik - aber zugleich auch zur Verkörperung einer lebensfernen, verschwommenen Kunstauffassung. Diese Einführung in Leben und Werk stellt deshalb zuerst den Menschen Friedrich von Hardenberg vor und verfolgt seine intensive Auseinandersetzung mit den politischen, philosophischen, naturwissenschaftlich-technischen, religiösen und ästhetischen Hauptfragen der Zeit zwischen 1789 und 1800. Erst auf diesem Hintergrund wird das lyrische, epische, (natur)philosophische und aphoristische Werk von Novalis untersucht und dessen Rezeptionsgeschichte beleuchtet.

Mit der Veröffentlichung des gesamten Jugendnachlasses Friedrich von Hardenbergs, den Hans-Joachim Mähl im Jahr 1983 in Krakau wiederentdeckt und 1998-99 in Zusammenarbeit mit Martina Eicheldinger herausgegeben hatte, wurde ein Signal gesetzt, es sei nun Zeit, nicht nur diese Bände der Historisch-kritischen Novalis-Ausgabe, sondern auch die sowohl qualitativ als auch quantitativ beachtliche Menge an Forschungsliteratur zu berücksichtigen, die seit Herbert Uerlings 
mittlerweile zum Standardwerk der Novalisforschung gewordener Habilitationsschrift, Friedrich von Hardenberg, genannt Novalis. Werk und Forschung (Stuttgart 1991), erschienen ist. Auch die Gründung der Internationalen Novalis-Gesellschaft im Jahr 1992 hat befruchtend auf den Fortgang der Forschung gewirkt; so war es mir z.B. noch möglich, die von Herbert Uerlings herausgegebenen Ergebnisse der zweiten Fachtagung dieser Gesellschaft - "Blüthenstaub«. Rezeption und Wirkung des Werkes von Novalis (Tübingen 2000) - im letzten Kapitel dieser Arbeit zu verarbeiten. Die Angaben zur herangezogenen Sekundärliteratur sind in der Bibliographie den jeweiligen Kapiteln im Haupttext zugeordnet, um es Studierenden zu erleichtern, die einschlägige Forschung zu bestimmten Werken gebündelt nachzuschlagen.

Danken möchte ich neben Kollegen an der Universität Vermont, die Interesse gezeigt und mich während der Arbeit an diesem Projekt immer wieder ermuntert haben, auch der Universität Vermont selbst und deren "University Committee for Research and Scholarship", die mich durch ein Forschungsjahr und ein Reisestipendium zum Besuch des Freien Deutschen Hochstifts in Frankfurt am Main und dem Geburtsort Friedrich von Hardenbergs in Schloss Oberwiederstedt, Sachsen-Anhalt, unterstützten. Von den beiden letztgenannten Einrichtungen waren sowohl Renate Moering, Leiterin der HandschriftenAbteilung, und ihr Kollege, Hans Grüters, als auch Gabriele Rommel, Leiterin der Forschungsstätte für Frühromantik und Novalis-Museum, und ihre Sekretärin, Petra Sehnert, besonders entgegenkommend in ihrer Hilfe. Näher zu Hause gilt mein Dank der Fernleihe der Universitätsbibliothek Vermont sowie der sachkundigen Hilfe des Referenzspezialisten Jake Barickman, die meine Forschungen entscheidend erleichtert haben. Dadurch, dass Sophia Vietor mir das Manuskript zu ihrer Studie "Astralisu von Novalis. Handschrift - Text - Werk (Würzburg 2001) zur Verfügung stellte, konnte ich auch diese bedeutende Neuveröffentlichung verwerten. Ebenfalls hilfreich in dieser Hinsicht waren Andreas Greiner und Gerhard Schulz, die mir freundlicherweise die Kopien einer gerade erschienenen Lesung des 5. Kapirels von Heinrich von Ofterdingen (CD-ROM-Aufnahme im Freiberger Stadt- und Bergbaumuseum, mit Begleitmusik auf dem sich dort befindlichen Orgelpositiv) bzw. einer Auswahl von Texten Novalis. Über die Liebe (Frankfurt a.M. 2001) zugestellt haben. Danken möchte ich weiterhin Herbert Uerlings, Sophia Vietor und David Wood für die kritische Lektüre einzelner Teile meines Manuskripts - aber insbesondere meiner geliebten Frau Angelika Mahoney und meinem geschätzten Freund Wolfgang Mieder, die das ganze Buch von Anfang bis Ende gelesen und mich immer mit Wort und Tat unterstützt haben. 
Am 31. März 2001 - sechs Tage nach dem 200. Todestag Friedrich von Hardenbergs und einen Tag, nachdem ich mein Buchmanuskript abgeschickt hatte - ist Hans-Joachim Mähl, der erste Präsident der Internationalen Novalis-Gesellschaft, gestorben. Was Hans-Joachim Mähls Veröffentlichungen und editorische Tätigkeit für die NovalisForschung des 20. Jahrhunderts bedeuten, braucht nicht hervorgehoben zu werden: Diese Leistungen sprechen für sich selber. Wer das Glück hatte, ihn kennen gelernt oder Briefe mit ihm gewechselt zu haben, wird den persönlichen Verlust spüren, den so viele jetzt empfinden. Ich hätte ihm so gerne ein Exemplar dieser Arbeit geschenkt.

Dieses Buch ist Melissa Mahoney gewidmet.

Burlington, Vermont im Juni 2001

Dennis F. Mahoney 


\section{Inhalt}

Vorwort $. . . \ldots \ldots \ldots \ldots \ldots \ldots \ldots \ldots \ldots \ldots, \mathrm{V}$

I. Herkunft, Kindheit und Jugend $\ldots \ldots \ldots \ldots \ldots \ldots 1$

1. Friedrich von Hardenberg, genannt Novalis .......... 1

2. Familie und Kindheit ........................ 2

3. Der Jugendnachlass und seine Bedeutung für das Gesamtwerk ...................... 6

II. Studienjahre in Jena, Leipzig und Wittenberg .... 11

1. Schöngeistige Lektüre als Vorbereitung auf das Studium .... 11

2. Lehrjahre in Jena: Studium bei Schiller und Reinhold ..... 12

3. Freundschaft mit Friedrich Schlegel und Liebesverwirrungen in Leipzig ............................ 16

4. Studienabschluss in Wittenberg und Sehnsucht nach $»$ Brautnacht" $\ldots \ldots \ldots \ldots \ldots \ldots \ldots \ldots \ldots \ldots, 21$

III. Berufliche Anfänge, Fichte-Studien und Sophie von Kühn .................... 25

1. Ausbildung in Tennstedt ...................... 25

2. Sophie von Kühn: »sittlicher Grazie / Vollendetes Bewußtseyn? $\ldots \ldots \ldots \ldots \ldots \ldots \ldots \ldots \ldots \ldots \ldots \ldots . \ldots \ldots$

3. Zusammenarbeit mit dem Vater; Einwilligung zur Verlobung ........................... 31

4. Erneuter Kontakt mit Friedrich Schlegel; Die Fichte-Studien........................ 34

5. Letzte Krankheit und Tod Sophie von Kühns.......... 41

IV. 1797: „Die Geburtsstunde des Romantikers«...... 44

1. Von Hardenberg zu $»$ Novalis« ................. 44

2. Der Grabbesuch vom 13. Mai 1797 :

Dichtung und Wahrheit................... 45 
3. Sprachschöpferische Dokumente: Hardenbergs Briefe und Journal vom Frühjahr $1797 \ldots \ldots \ldots \ldots$. . . . . 47

4. Neue Hinwendung zu den Wissenschaften:

Studien zu Hemsterhuis, Kant und Schelling.......... 50

V. Romantische Anfänge: Bergakademie und Blüthenstaub..................... 57

1. Studium an der Bergakademie Freiberg ........... 57

2. Reizmedizinische Poetik: „Construction der transscendentalen Gesundheit $\ldots \ldots \ldots \ldots \ldots \ldots 60$

3. Vermischte Bemerkungen / Blüthenstaub .............62 62

4. Goethe: „der wahre Statthalter des poetischen Geistes auf Erden"............................. 65

VI. Romantische Staatsphilosophie: Glauben und Liebe ..................... 69

1. "Novalis« und Hoffnung auf 'Neuland in Preußen ...... 69

2. Der poetische Staat ..................... 72

3. "Tropen und Räthselsprache" .................. 73

4. Wirkungsgeschichte $\ldots \ldots \ldots \ldots \ldots \ldots \ldots \ldots \ldots \ldots \ldots \ldots \ldots$

VII. Naturphilosophie und Enzyklopädistik ........ 81

1. Das Allgemeine Brouillon .................. 81

1.1 "Die Religion des sichtbaren Weltalls" .......... 81

1.2 Das Allgemeine Brouillon: ,Entwurf, oder $\cdot$ Rührey? . . . . . . . . . . . . . . . . . 83

1.3 Wirkungsgeschichte - geschichtliches Wirken ..... 86

2. Die Lehrlinge $z u$ Sais ..................... 89

2.1 Die Lehrlinge zu Sais: ,Mischung, oder ,Gemisch?? . . 89

2.2 "Der Lehrling«: Romantische Figurenlehre........ 92

2.3 „Die Natur «: Auditive Ästherik................ 94

2.4 Das Märchen von Hyazinth und Rosenblüthe ........ 97

2.5 Das Märchen als Bruchstück einer sscientifischen Bibel........................... 99

2.6 „ÜbergangsJahre vom Unendlichen zum Endlichen ${ }^{2} \ldots \ldots \ldots \ldots \ldots \ldots \ldots \ldots$ 
VIII. Romantische Geschichtsphilosophie:

Die Christenheit oder Europa................ 103

1. "Cosmopoliten Loge" und "Atheismusstreit" . . . . . . . 103

2. Romantisierte Religion .................... 105

3. Öffentliche Rede, Poesie oder Predigt? ............ 108

4. Geschichts-, Religions- und Naturphilosophie ......... 110

5. Editions- und Wirkungsgeschichte ............. 112

IX. Exoterisches und esoterisches Christentum ..... 115

1. Geistliche Lieder......................... 115

1.1 "Probe eines neuen geistlichen Gesangbuchs" ..... 115

1.2 „Wenige wissen / Das Geheimniß der Liebe" ...... 119

2. Hymnen an die Nacht ..................... 121

2.1 "Nachtbegeisterung $\ldots \ldots \ldots \ldots \ldots \ldots \ldots \ldots \ldots, 121$

2.2 „Neue Mythologie«? . . . . . . . . . . . . . . . 124

X. Heinrich von Ofterdingen ............... 126

1. Entstehungsgeschichte und Gesamtkonzeption......... 126

2. Wilhelm Meisters Lehrjahre: Kritik und Aneignung ...... 131

3. "Das Klingsohr-Märchen «................... 134

4. Eine "Erfüllung«, die "Erwartung" blieb........... 136

XI. Aussichten - auch in die Ewigkeit . . . . . . . . . 139

1. Berufliche und familiäre Hoffnungen .............. 139

2. Die späten Gedichte, Aufzeichnungen und schriftstellerischen Pläne ................. 142

3. Letzte Krankheit und Tod ................. 145

XII. Abschließendes zur Rezeption ............ 147

XIII. Bibliographie ....................... 155

Personenregister $\ldots \ldots \ldots \ldots \ldots \ldots \ldots \ldots \ldots \ldots \ldots$ 